\title{
RANDOM MAPPINGS WITH A SINGLE ABSORBING CENTER AND COMBINATORICS OF DISCRETIZATIONS OF THE LOGISTIC MAPPING ${ }^{1}$
}

\author{
A. KLEMM \\ Deakin University \\ School of Computing and Mathematics \\ Geelong, Vic 3217, Australia \\ A. POKROVSKII ${ }^{2}$ \\ National University of Ireland \\ Institute for Nonlinear Science, Department of Physics \\ University College, Cork, Ireland \\ E-mail: alexei@peterhead.ucc.ie
}

(Received November, 1997; Revised December, 1998)

\begin{abstract}
Distributions of basic characteristics of random mappings with a single absorbing center are calculated. Results explain some phenomena occurring in computer simulations of the logistic mapping.

Key words: Chaotic Numerics, Logistic Mapping, Discretization, Random Graphs.

AMS subject classifications: 34 A50, B58F13.
\end{abstract}

\section{Introduction}

Analysis of combinatorical properties of discretizations of dynamical systems constitutes a new challenging and important area. In this area a special spot is occupied by analysis of discretizations of the logistic mapping

$$
f(x)=4 x(1-x), \quad x \in[0,1] .
$$

${ }^{1}$ This research has been supported by the Australian Research Council Grant A 89132609 .

${ }^{2}$ Permanent address: Institute of Information Transmission Problems Russian Academy of Science, Moscow. This paper was initially written when Pokrovskii was working at Deakin University and was supported by School of Computing and Mathematics. 
The reason is that although this mapping is the very simplest example of continuous mapping with quasi-chaotic behavior $[9,11]$, nevertheless, its discretizations demonstrate unexpected behavior in many respects [3]. In particular, the methods suggested in [10] and refined in [8] are not adequate in investigating the mapping (1.1). In this paper we will show how some properties of discretizations of the logistic mapping can be explained on the basis of properties of a special family of random mappings. This paper extends an approach suggested in [6].

Other related studies include research on period lengths of one-dimensional discretized systems carried out in detail by Beck $[1,2]$, similar questions with different maps by Percival and Vivaldi [13], and some general questions concerning noisy orbits by Nusse and Yorke [12].

\section{Auxiliary Notations}

In this paper $\mathbb{R}^{N}$ denotes the $N$-dimensional coordinate Euclidean space; elements from $\mathbb{R}^{N}$ will be denoted by $\mathbf{x}=\left(x^{1}, \ldots, x^{N}\right)$. Let

$$
Q^{N}=\left\{\left(x^{1}, \ldots, x^{N}\right): 0 \leq x^{n} \leq 1, n=1, \ldots, N\right\}
$$

be the unit cube in $\mathbb{R}^{N}$. For each $N$ denote by $\mu^{N}$ the product measure over $Q^{N}$ with identical absolutely continuous coordinate probability measures $\mu$.

We will be interested in limit behavior as $N \rightarrow \infty$ of measures $\mu^{N}\left(S^{N}\right)$ of some special sets $S^{N}$ which are described in this paragraph. Denote $r_{0}\left(x^{1}\right) \equiv 1$ and define inductively sequences of functions by

$$
\begin{gathered}
r_{N}\left(x^{1}, \ldots, x^{N}\right)=\prod_{i=1}^{N}\left(1-x^{i}\right), \quad N=1,2, \ldots \\
y_{N}\left(x^{1}, \ldots, x^{N}\right)=r_{N-1}\left(x^{1}, \ldots, x^{N-1}\right) x^{N}, \quad N=1,2, \ldots
\end{gathered}
$$

Finally, denote

$$
Y^{N}(\mathbf{x})=\left\{y_{1}\left(x^{1}\right), y_{2}\left(x^{1}, x^{2}\right), \ldots, y_{N}\left(x^{1}, \ldots, x^{N}\right)\right\} \quad N=1,2, \ldots
$$

Now define by ord ${ }_{k}\left(y_{1}, y_{2}, \ldots, y_{n}\right)$ the decreasing sequences of the $k$ largest elements of the finite (ordered or unordered) set $\left\{y_{1}, y_{2}, \ldots, y_{n}\right\}$ and let

$$
Y_{k}^{N}(\mathbf{x})=\operatorname{ord}_{k}\left(Y^{N}(\mathbf{x})\right)
$$

Define the functions

$$
\begin{gathered}
D_{k}^{N}(\mathbf{z} ; \mu)=\mu^{N}\left(\left\{\mathbf{x} \in Q^{N}: 0 \leq Y_{k}^{N}(\mathbf{x}) \leq \mathbf{z}\right\}\right), \quad \mathbf{z} \in Q^{k} \\
R^{N}(\alpha ; \mu)=\mu^{N}\left(\left\{\mathbf{x} \in Q^{N}: 0 \leq r_{N}(\mathbf{x}) \leq \alpha\right\}\right), \quad 0 \leq \alpha \leq 1
\end{gathered}
$$

where inequality is understood to be coordinate-wise.

Lemma 1: There exists the continuous uniform limit 


$$
F_{k}(\mathbf{z} ; \mu) \triangleq \lim _{N \rightarrow \infty} D_{k}^{N}(\mathbf{z} ; \mu), \quad \mathbf{z} \in Q^{k}
$$

and for each $N$ the estimates

are valid.

$$
D_{k}^{N}(\mathbf{z} ; \mu)-R_{1 \leq i \leq k}^{N}\left(\min _{1 \leq}\left\{z^{i}\right\} ; \mu\right) \leq F_{k}(\mathbf{z} ; \mu) \leq D_{k}^{N}(\mathbf{z} ; \mu), \quad \mathbf{z} \in Q^{k}
$$

Proof: By the definition, the set $Y^{N}(\mathbf{x})$ is a subset of $Y^{M}(\mathbf{x})$ for $N<M, \mathbf{x} \in Q^{M}$; therefore

and further

$$
Y_{k}^{N}(\mathbf{x}) \leq Y_{k}^{M}(\mathbf{x}), \quad N<M, \quad \mathbf{x} \in Q^{M}
$$

$$
\mu^{M}\left(\left\{\mathbf{x} \in Q^{M}: 0 \leq Y_{k}^{N}(\mathbf{x}) \leq \mathbf{z}\right\}\right) \geq \mu^{M}\left(\left\{\mathbf{x} \in Q^{M}: 0 \leq Y_{k}^{M}(\mathbf{x}) \leq \mathbf{z}\right\}\right) .
$$

On the other hand, $Y_{k}^{N}(\mathbf{x})$ depends only on the first $N$ coordinates of $\mathbf{x} \in Q^{M}$ and the measure $\mu^{M}$ is a coordinate probability measure, which yields

$$
\mu^{N}\left(\left\{\mathbf{x} \in Q^{N}: 0 \leq Y_{k}^{N}(\mathbf{x}) \leq \mathbf{z}\right\}\right)=\mu^{M}\left(\left\{\mathbf{x} \in Q^{M}: 0 \leq Y_{k}^{N}(\mathbf{x}) \leq \mathbf{z}\right\}\right)
$$

The last two displayed inequalities imply

$$
D_{k}^{N}(\mathbf{z} ; \mu) \geq D_{k}^{M}(\mathbf{z} ; \mu), \quad \mathbf{z} \in Q^{k}
$$

for each positive integer $M>N$ (note that the inequality sign has been reversed while converting (2.7) into (2.8)).

Denote by $\widehat{y}_{k}^{N}(\mathbf{x})$ the $k$-th largest element of the set $Y^{N}(\mathbf{x})$, that is the last coordinate of $Y_{k}^{N}(\mathbf{x})$. The inequality

implies

$$
r_{N}(\mathbf{x}) \leq \widehat{y}_{k}^{N}(\mathbf{x})
$$

$$
Y_{k}^{N}(\mathbf{x})=Y_{k}^{M}(\mathbf{x}), \mathbf{x} \in Q^{M}
$$

for all $M \geq N$ because all elements of the set $Y^{M}(\mathbf{x}) \backslash Y^{N}(\mathbf{x})$ are not greater than $r_{N}(\mathbf{x})$. In particular, for each $\mathbf{z} \in Q^{k}$ the following inclusion holds

$$
\begin{gathered}
\left\{\mathbf{x} \in Q^{M}: 0 \leq Y_{k}^{N}(\mathbf{x}) \leq \mathbf{z}\right\} \backslash\left\{\mathbf{x} \in Q^{M}: 0 \leq Y_{k}^{M}(\mathbf{x}) \leq \mathbf{z}\right\} \\
\subseteq\left\{\mathbf{x} \in Q^{M}: 0 \leq r_{N}(\mathbf{x}) \leq \min _{1 \leq i \leq k}\left\{z^{i}\right\}\right\} .
\end{gathered}
$$

On the other hand, $Y_{k}^{N}(\mathbf{x})$ depends only on the first $N$ coordinates of $\mathbf{x} \in Q^{M}$ and the measure $\mu^{M}$ is a coordinate probability measure, which yields

$$
\mu^{N}\left(\left\{\mathbf{x} \in Q^{N}: 0 \leq Y_{k}^{N}(\mathbf{x}) \leq \mathbf{z}\right\}\right)=\mu^{M}\left(\left\{\mathbf{x} \in Q^{M}: 0 \leq Y_{k}^{N}(\mathbf{x}) \leq \mathbf{z}\right\}\right) .
$$

The last two displayed inequalities imply

$$
D_{k}^{N}(\mathbf{z} ; \mu) \geq D_{k}^{M}(\mathbf{z} ; \mu), \quad \mathbf{z} \in Q^{k}
$$


for each positive integer $M>N$ (note that the inequality sign has been reversed while converting (1.7) into (1.8)).

Denote by $\widehat{y}_{k}^{N}(\mathbf{x})$ the $k$-th largest element of the set $Y^{N}(\mathbf{x})$, that is the last coordinate of $Y_{k}^{N}(\mathbf{x})$. The inequality

implies

$$
r_{N}(\mathbf{x}) \leq \widehat{y}_{k}^{N}(\mathbf{x})
$$

$$
Y_{k}^{N}(\mathbf{x})=Y_{k}^{M}(\mathbf{x}), \quad \mathbf{x} \in Q^{M}
$$

for all $M \geq N$ because all elements of the set $Y^{M}(\mathbf{x}) \backslash Y^{N}(\mathbf{x})$ are not greater than $r_{N}(\mathbf{x})$. In particular, for each $\mathbf{z} \in Q^{k}$ the following inclusion hold

and, further,

$$
\begin{gathered}
\left\{\mathbf{x} \in Q^{M}: 0 \leq Y_{k}^{N}(\mathbf{x}) \leq \mathbf{z}\right\} \backslash\left\{\mathbf{x} \in Q^{M}: 0 \leq Y_{k}^{M}(\mathbf{x}) \leq \mathbf{z}\right\} \\
\subseteq\left\{\mathbf{x} \in Q^{M}: 0 \leq r_{N}(\mathbf{x}) \leq \min _{1 \leq i \leq k}\left\{z^{i}\right\}\right\}
\end{gathered}
$$

$$
D_{k}^{M}(\mathbf{z} ; \mu) \geq D_{k}^{N}(\mathbf{z} ; \mu)-R^{N}\left(\min _{1 \leq i \leq k}\left\{z^{i}\right\} ; \mu\right), \quad \mathbf{z} \in Q^{k}
$$

Combining (1.8) and (1.10) yields

$$
D_{k}^{N}(\mathbf{z} ; \mu)-R^{N}\left(\min _{1 \leq i<k}\left\{z^{i}\right\} ; \mu\right) \leq D_{k}^{M}(\mathbf{z} ; \mu) \leq D_{k}^{N}(\mathbf{z} ; \mu), \quad \mathbf{z} \in Q^{k}
$$

for all $M \geq N$.

Because of continuity of the measure $\mu$ for each positive $\varepsilon$

$$
\lim _{N \rightarrow \infty} \sup _{\alpha \geq \varepsilon} R^{N}(\alpha ; \mu)=0, \varepsilon>0 \text { and } \lim _{\varepsilon \rightarrow 0} \sup _{\min \left\{z^{(i)}\right\} \leq \varepsilon} D_{k}^{N}(\mathbf{z} ; \mu)=0 .
$$

Thus (1.10) implies both assertions of the lemma.

Let $Z$ be the set of all finite sequences with the sum 1 . Let $g(\mathbf{z})$ be the nonnegative scalar function on $Z$ with the following properties:

(a) $g$ is symmetric, that is, the value $g(z)$ does not change under permutations of coordinates of $\mathbf{z} \in Z$;

(b) the value $g(z)$ does not change if we add to $\mathbf{z}$ some zero coordinates;

(c) if $\mathbf{z}_{1}$ is longer than $\mathbf{z}_{2}$ and they coincide for all coordinates but one then $g\left(\mathbf{z}_{1}\right) \leq g\left(\mathbf{z}_{2}\right)$.

Examples are given by the maximal coordinate, the second maximal coordinate, the sum of squares of coordinates

and many others.

$$
g_{*}(\mathbf{z})=\sum_{i}\left(z^{i}\right)^{2}
$$

Introduce the functions

$$
\begin{aligned}
& D_{g}^{N}(\alpha ; \mu)=\mu^{N}\left(\left\{\mathbf{x} \in Q^{N}: g\left(Y^{N}(\mathbf{z})\right) \leq \alpha\right\}\right) \\
& \underline{D}_{g}^{N}(\alpha ; \mu)=\mu^{N}\left(\left\{\mathbf{x} \in Q^{N}: g\left(Y_{*}^{N}(\mathbf{x})\right) \leq \alpha\right\}\right)
\end{aligned}
$$


where

$$
Y_{*}^{N}(\mathbf{x})=\left\{y_{1}\left(x^{1}\right), y_{2}\left(x^{1}, x^{2}\right), \ldots, y_{N}\left(x^{1}, \ldots, x^{N}\right), r_{n}(\mathbf{x})\right\}
$$

Lemma 2: There exist the continuous uniform limit

$$
F_{g}(\alpha ; \mu) \triangleq \lim _{N \rightarrow \infty} D_{g}^{N}(\alpha ; \mu)
$$

and for each $N$ the estimates

$$
\underline{D}_{g}^{N}(\alpha ; \mu) \leq F_{g}(\alpha ; \mu) \leq D_{g}^{N}(\alpha ; \mu)
$$

are valid.

Proof: This follows the same arguments as the proof of the previous lemma and so is omitted.

This lemma is effective as a tool for numerical computation of the corresponding limit functions. Consider the case when the measure $\mu$ is given by the distribution function

$$
\mu([0, x])=1-\sqrt{1-x}
$$

which will be important in the next section. Here usually the gap between upper and lower estimates is of the magnitude $10^{-3}$ for $N=3$ and decreases very fast in $N$. Figure 1 graphs the limit functions $F_{1}(\alpha ; \mu)$ and $F_{g_{*}}(\alpha ; \mu)$ against the distribution (1.17).

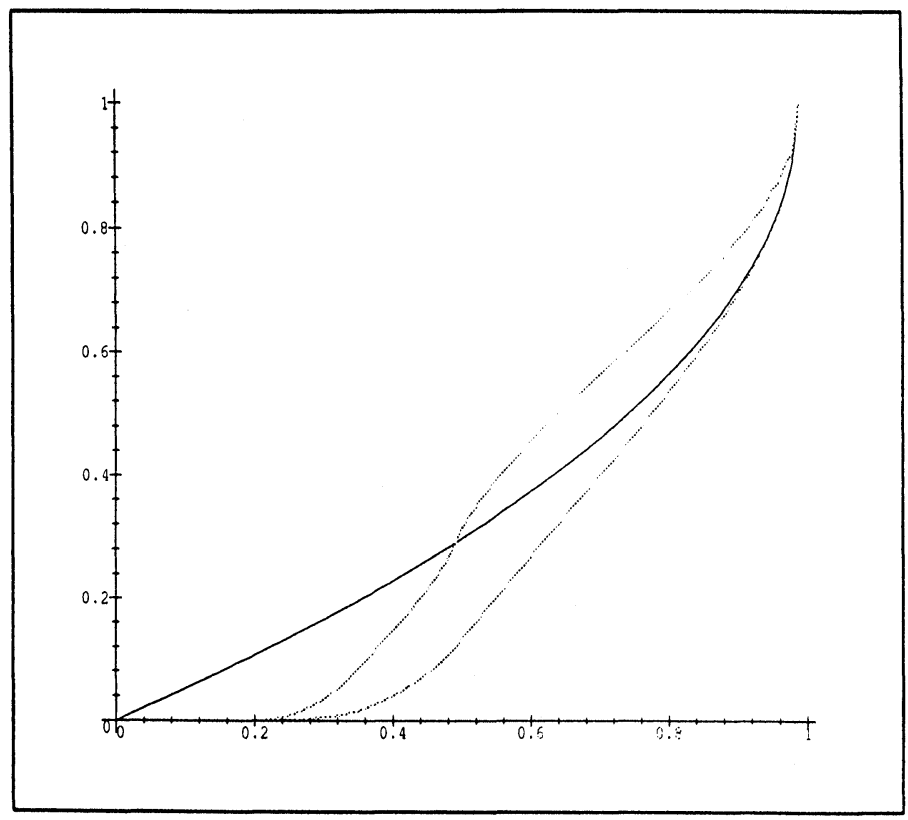

Figure 1. The limit functions $F_{1}(z ; \mu), F_{g_{*}}(z ; \mu)$ and the distribution $\mu([0, z])$ $=1-\sqrt{1-z}$ (bold). 


\section{Random Mappings with a Single Absorbing Center}

Let $\Delta, K>0$ be positive integers and let

$$
X(\Delta, K)=\{-\Delta+1, \ldots,-1,0,1, \ldots, K\} .
$$

Define the set $\Psi(\Delta, K)$ of all mappings $\psi: X(\Delta, K) \rightarrow X(\Delta, K)$ satisfying $\psi(i)=0$ for $i \leq 0$. This collection is called a random mapping, with an absorbing center. The set $\{\xi \in X(\Delta, K): x \leq 0\}$ is the absorbing center; once a trajectory of $\psi$ enters this set it cannot leave. If $S$ is a subset of $\Psi(\Delta, K)$, associated with some given property $A$, then the proportion of elements of $\Psi$ which belong to $S$ will be called the probability of the event $A$ and is denoted by $P(A ; \Psi(\Delta, K))$.

Random mappings with an absorbing center are similar to mappings with a single attracting center $[4,5,15]$.

\subsection{Distributions of Basins of Attractions}

For each $\psi \in \Psi(\Delta, K)$ the set $X(\Delta, K)$ is partitioned naturally into a disjoint union of basins of attractions of different cycles of the mapping $\psi$. Denote by $\mathscr{B}(\psi)$ the set of cardinalities of basins of attractions and

$$
\mathscr{B}_{k}(\psi)=\operatorname{ord}_{k}(\mathscr{B}(\psi))
$$

(here the $i$-th element of $\operatorname{ord}_{k}(\mathscr{B}(\psi))$ is defined as zero if $i$ is greater than the total number of different basin of attractions). Recall that the distribution function of the finite set $S \subset Q^{k}$ is defined as

$$
\mathscr{D}(\mathbf{z} ; S)=\frac{\aleph(\{\mathbf{u} \in S: \mathbf{u} \leq \mathbf{z}\})}{\aleph(S)}
$$

for $\mathbf{z} \in Q^{k}$ (here and below $\aleph(S)$ denotes the cardinality of the set $S$ ). Denote

$$
D_{\mathscr{B}, k}(\mathbf{z} ; \Delta, K)=\mathscr{D}\left(\mathbf{z} ;\left\{\frac{\mathscr{B}_{k}(\psi)}{\Delta+K}: \psi \in \Psi(\Delta, K)\right\}\right)
$$

and

$$
\mathbf{y}_{k}(\mathbf{z}, c)=\max \left\{\mathbf{y} \in Q^{k}: \operatorname{ord}_{k}(\mathbf{y}, c) \leq \mathbf{z}\right\}
$$

Proposition 1: The limit equality

$$
\lim _{n \rightarrow \infty} D_{\mathscr{B}, k}(\mathbf{z} ;[a n],[b \sqrt{n}])=\int_{0}^{1} F_{k}\left(\mathbf{y}\left(\frac{\mathbf{z}}{1-s}\right) ; \mu\right) d \gamma_{c}(s)
$$

holds, where $c=a / \sqrt{b},[\cdot]$ is the floor function $\gamma_{c}(x)=\operatorname{ERFC}\left(c \sqrt{\frac{1-x}{2 x}}\right)$ and

$$
\operatorname{ERFC}(t)=\frac{2}{\sqrt{\pi}} \int_{t}^{\infty} e^{-s^{2}} d s
$$

is the complimentary error function.

The proof is relegated to the next section. By this proposition, only the value $c=a / \sqrt{b}$ influences the limit behavior of distributions $D_{\mathscr{B}, k}(\mathbf{z} ;[a n],[b \sqrt{n}])$ as $n \rightarrow \infty$. 
This value $c$ is called the absorbing coefficient.

In particular, consider the case $k=1$, that is the limit behavior of the largest basin of attraction. Introduce functions

for $0 \leq x, y \leq 1$ and

$$
F(x, y ; c)=\int_{0}^{x} F_{1}\left(\frac{y}{1-s} ; \mu\right) d \gamma_{c}(s)
$$

$$
F_{\mathfrak{B}, 1}(x ; c)=\left\{\begin{array}{cl}
F(x, x ; c), & \text { if } 0 \leq x \leq 1 / 2, \\
F(1-x, x ; c)+\gamma_{c}(x)-\gamma_{c}(1-x), & \text { if } 1 / 2 \leq x \leq 1
\end{array}\right.
$$

\section{Corollary 1:}

$$
\lim _{n \rightarrow \infty} D_{\mathscr{B}, 1}(\alpha ;[a \sqrt{n}],[b n])=F_{\mathscr{B}, 1}(\alpha ; a / \sqrt{b}) .
$$

It is instructional that even rather small values of the absorbing coefficient $c=a / \sqrt{b}$ influence significantly and in nonevident manner the behavior of the corresponding limit distributions. Figure 2 graphs the case $c=0$, that is the function from the previous section against the cases $c=0.25, c=1$ and $c=2$. Clearly the weak absorbing center $c=0.25$ increases significantly the corresponding distribution function whereas the strong absorbing center $c=2$ decreases it.

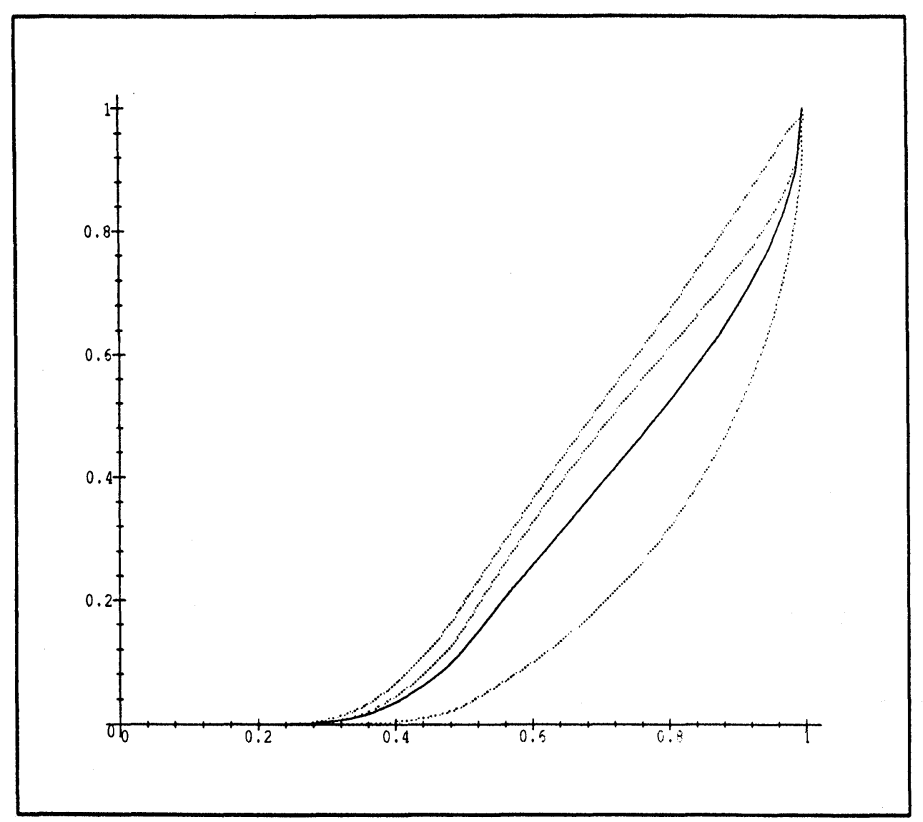

Figure 2. Limit distributions $F_{\mathscr{B}, 1}(\alpha ; a / \sqrt{b})$ of maximal basin distributions for $a / \sqrt{b}=0$ (bold) and for $a / \sqrt{b}=0.25,1.0,2.0$ (from above). 
Consider for each $\psi$ the sum of squares

$$
g_{*}(\psi)=\sum_{\beta \in \mathscr{B}(\psi)}\left(\frac{\beta}{\Delta+K}\right)^{2} .
$$

This characteristic is especially important because this estimates the probability that two random points from $X(\Delta, K)$ generate the same cycle. Analogous to the previous corollary the following can be established.

\section{Corollary 2:}

$$
\lim _{n \rightarrow \infty} D_{g_{*}}(\alpha ;[a \sqrt{n}],[b n])=F_{g_{*}}(\alpha ; a / \sqrt{b})
$$

where $g_{*}(\cdot)$ is the above sum of squares and

$$
F_{g_{*}}(\alpha ; c)=\int_{0}^{\sqrt{a}} F_{g_{*}}\left(\frac{\alpha-s^{2}}{(1-s)^{2}} ; \mu\right) d \gamma_{c}(s) .
$$

Figure 3 is analogous to Figure 2 but deals with the limit distribution of sum of squared basins of attraction.

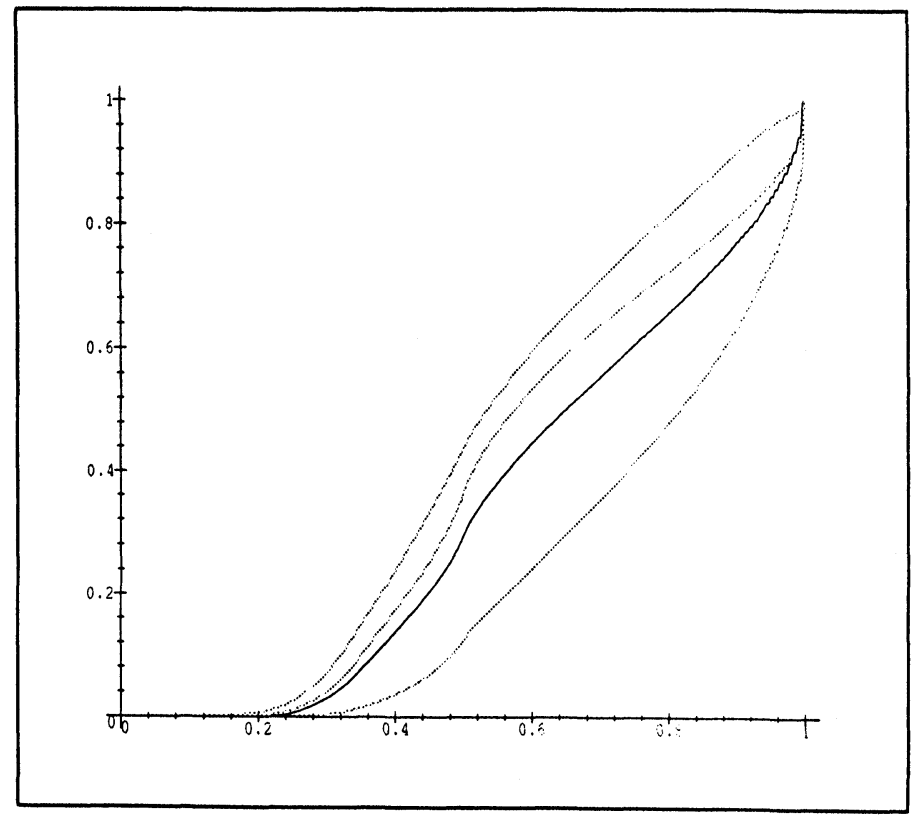

Figure 3. Limit distributions $F_{g_{*}}(\alpha ; a / \sqrt{b})$ of squared basin distributions for $a / \sqrt{b}=0$ (bold) and for $a / \sqrt{b}=0.25,1.0,2.0$ (from above).

Let us mention on simple explicit formula in this direction. Denote by $\mathscr{B}(i, \psi)$ the cardinality of the basin of attraction which contains a particular element $i \in X(\Delta, K)$ 
and introduce the function

$$
D_{\mathscr{B}}(\alpha ; \psi)=\mathscr{T}\left(\alpha ;\left\{\frac{\mathscr{B}(i ; \psi)}{\Delta+K}: i \in X(\Delta, K)\right\}\right) .
$$

We emphasize that this characteristic is a scalar function on $[0,1]$. Denote finally

$$
M_{\mathscr{B}}(\alpha ; \Delta, K)=\frac{1}{\aleph(\Psi(\Delta+K))} \sum_{\psi \in \Psi(\Delta, K)} D_{\mathscr{B}}(\alpha ; \psi) .
$$

That is the mean value of functions $D_{\mathscr{B}}(\alpha ; \psi)$ over $\psi \in \Psi(\Delta, K)$.

Proposition 2: There exists the uniform limit

$$
\lim _{n \rightarrow \infty} M_{\mathscr{B}}(\alpha ;[a \sqrt{n}],[b n])=F_{\mathscr{B}}(\alpha ; a / \sqrt{b})
$$

with

$$
\begin{gathered}
F_{\mathfrak{B}}(\alpha ; c)=\alpha \gamma_{c}(\alpha)+\int_{\alpha}^{1} \gamma_{c}(\theta) d \theta \\
\left.-\int_{\alpha}^{1} \gamma_{c}(1-\theta) \sqrt{\theta-\alpha} d \sqrt{\theta}+\sqrt{\theta} d \sqrt{\theta-\alpha}\right) .
\end{gathered}
$$

Figure 4 is analogous to Figures 2 and 3; this figure graphs mean distributions for the given parameter values.

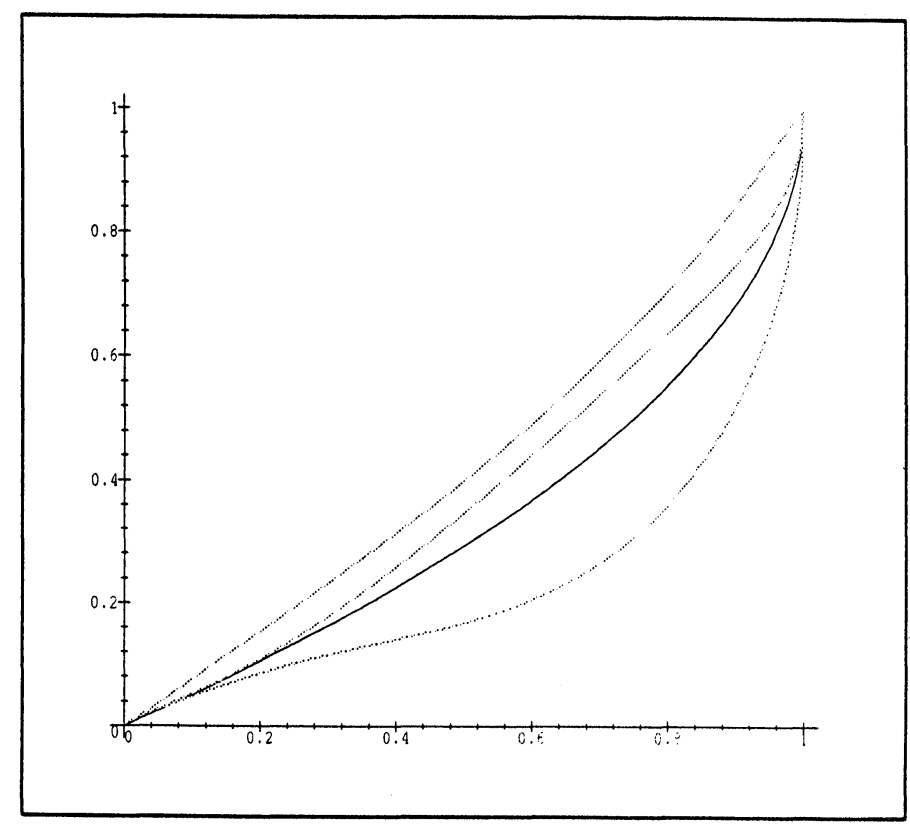

Figure 4. Limit distributions $F_{\mathscr{B}}(\alpha ; a / \sqrt{b})$ of averaged basin distributions for $a / \sqrt{b}=0$ (bold) and for $a / \sqrt{b}=0.25,1.0,2.0$ (from above). 


\subsection{Distribution of the Cycle Lengths}

Denote by $\mathfrak{C}(\psi)$ the set to cycle lengths of the mapping $\psi$ and denote

Introduce the distribution function

$$
\mathrm{e}_{k}(\psi)=\operatorname{ord}_{k}(\mathrm{e}(\psi))
$$

$$
D_{\mathcal{C}_{, k}}(\mathbf{z} ; \Delta, K)=\mathscr{D}\left(\mathbf{z} ;\left\{\frac{\mathrm{e}_{k}(\psi)}{\sqrt{\Delta+K}}: \psi \in \Psi(\Delta, K)\right\}\right) .
$$

where the operation $\mathscr{D}$ is defined as in (2.1) with the difference that $\mathbf{z}$ belongs to the set of $k$-dimensional vectors with nonnegative components. In line with Proposition 1 , it can be proved.

Proposition 3: There exist the uniform limits

$$
F_{\mathrm{C}, k}(\mathbf{z} ; a, b) \triangleq \lim _{n \rightarrow \infty} D_{\mathrm{C}, k}(\mathbf{z} ;[a, \sqrt{n}],[b n]),
$$

where the equality

with

$$
F_{\mathrm{C}, k}(\mathbf{z} ; a, b)=F_{\mathrm{C}, k}^{*}(\sqrt{b} \mathbf{z} ; a / \sqrt{b})
$$

$$
F_{\mathrm{C}, k}^{*}(\mathbf{z} ; c)=\int_{0}^{1} F_{\mathrm{e}, k}\left(\frac{\mathbf{z}}{\sqrt{1-s}} ; 0,1\right) d \gamma_{c}(s)
$$

holds.

In particular, consider the case $k=1$. By the Stepanov formula

$$
F_{\mathrm{C}, 1}(\alpha ; 0,1) \triangleq S(\alpha)=\frac{1}{\sqrt{2 \pi}} \int_{1-i \infty}^{1+i \infty} e^{-E(\alpha p)+p^{2} / 2} d p
$$

with

$$
E(x)=\int_{x}^{\infty} \frac{e^{-t}}{t} d t
$$

see [14], Formula (16), item 9, p. 919 (note that " $i$ " in front of the integral in this formula is a misprint). So Proposition 3 above implies

Corollary 3:

$$
F_{\mathrm{C}, 1}(\alpha ; a, b)=F_{\mathrm{C}, 1}^{*}(\sqrt{b} \alpha ; a / \sqrt{b})
$$

where

$$
F_{\mathrm{C}, 1}^{*}(\alpha, c)=\int_{0}^{1} S\left(\frac{\alpha}{\sqrt{1-s}}\right) d \gamma_{c}(s)
$$

See Figure 5 for the behavior of the functions $F_{\mathcal{C}, 1}^{*}(\alpha, c)$ for different $c$; here the influence of $c$ is "monotone" in contrast to the previous three figures. 


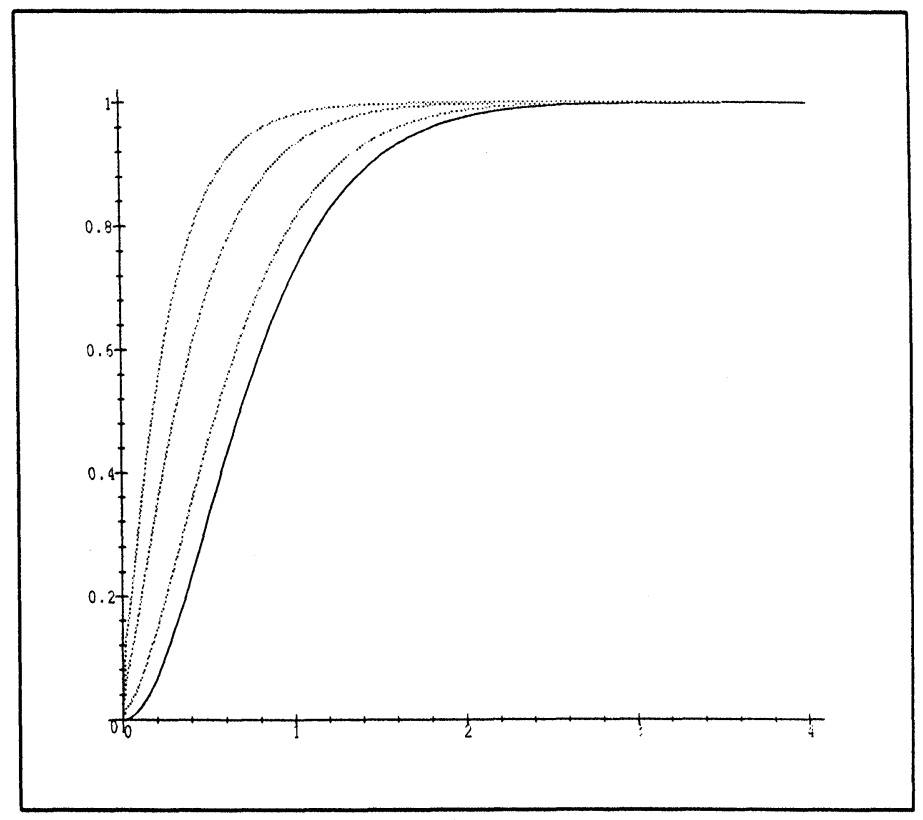

Figure 5. Limit distributions of normed maximal cycle length $b \equiv 1$ and for $a / \sqrt{b}=0,0.25,1.0,2.0$ (from below in this order).

Denote by $\mathfrak{C}(i ; \psi), i \in X(\Delta, K)$ the length of the unique cycle which is generated by a particular element $i \in X(\Delta, K)$ and

Denote finally

$$
D_{\mathrm{e}}(\alpha ; \psi)=\mathscr{I}\left(\alpha ;\left\{\frac{\mathrm{C}(i ; \psi)}{\sqrt{n}}: i \in X(\Delta, K)\right\}\right) .
$$

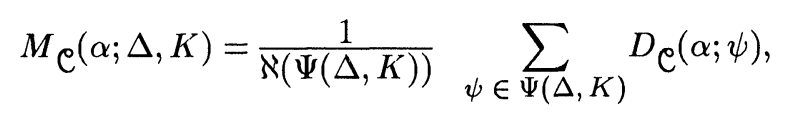

the mean value of functions $D_{\mathbb{C}}(\alpha ; \psi)$ over $\psi \in \Psi(\Delta, K)$.

Proposition 4: There exists the uniform limit

$$
F_{\mathrm{e}}(\alpha ; a, b) \triangleq \lim _{n \rightarrow \infty} M_{\mathrm{e}}(\alpha ;[a \sqrt{n}],[b n]), \quad x>0
$$

where the equality

$$
F_{\mathrm{e}}(\alpha ; a, b)=F_{c}^{*}(\alpha \sqrt{b} ; a / \sqrt{b})
$$

holds with

$$
F_{\mathrm{e}}^{*}(\alpha ; c)=1-e^{-\alpha\left(\frac{\alpha}{2}+c\right)}+\sqrt{\frac{\pi}{2}} e^{c^{2}}(\alpha+c) \operatorname{ERFC}\left(\frac{\alpha+c}{\sqrt{2}}\right)
$$




\subsection{Proof of Proposition 1}

It is convenient to define $\Psi(0, K)$ as a completely random mapping on the set

$$
X(0, K)=\{1, \ldots, K\}
$$

that is, as the totality of all possible mappings $X(0, K) \rightarrow X(0, K)$. Denote by $\beta(1, \psi)$ the cardinality of a basin of attraction which contains the element 1 and $r(1, \psi)=$ $K-\beta(1, \psi)$.

Lemma 3: The limit equality

$$
\lim _{n \rightarrow \infty} P\left(\frac{r(1, \psi)}{K} \leq \alpha: \Psi(0, K)\right)=\mu([\alpha, 1])=\sqrt{1-\alpha}
$$

is valid.

Proof: This follows from the Stepanov assertion [14], Corollary 1, p. 625.

Denote by $\widetilde{\beta}(i, \psi)$ the basin of attraction of the mapping $\psi$ which contains $i$ (not the cardinality of this basin as above!). Define by induction

$$
\widetilde{\beta}_{1}(\psi)=\widetilde{\beta}(1 ; \psi), \quad \widetilde{\beta}_{k}(\psi)=\widetilde{\beta}\left(i_{k} ; \psi\right)
$$

where $i_{k}$ is the minimal element which does not belong to the previous sets $\tilde{\beta}$. Finally denote, $\left.\beta_{k}(\psi)=\aleph\left(\widetilde{\beta}_{k}\right)\right)$ and $r_{k}(\psi)=r_{k-1}(\psi)-\beta_{k}(\psi)$.

Lemma 4: The limit equality

is valid.

$$
\lim _{n \rightarrow \infty} P\left(\frac{r_{k}(\psi)}{r_{k-1}(\psi)} \leq \alpha ; \Psi(0, K)\right)=\sqrt{1-\alpha}
$$

Proof: From the previous lemma by induction.

Let

and

$$
Y^{N}(\psi)=\left\{\frac{\beta_{1}(\psi)}{K}, \ldots, \frac{\beta_{N}(\psi)}{K}\right\}
$$

$$
Y_{k}^{N}(\psi)=\operatorname{ord}_{k}\left(Y^{N}(\psi)\right)
$$

\section{Corollary 4:}

$$
\lim _{n \rightarrow \infty} P\left(Y_{k}^{N}(\psi) \leq \mathbf{z} ; \Psi(0, n)\right) \triangleq D_{k}^{N}(\mathbf{z})=D_{k}^{N}(\mathbf{z} ; \mu) .
$$

This together with Lemma 1 gives

\section{Corollary 5:}

$$
\lim _{n \rightarrow \infty} D_{\mathscr{B}, k}(\mathbf{z}, 0, n)=\lim _{N \rightarrow \infty} D_{k}^{N}(\mathbf{z})=F_{k}(\mathbf{z} ; \mu) .
$$

Denote by $\beta(0, \psi)$ the cardinality of the points which are eventually absorbed by zero.

Lemma 5:

$$
\lim _{n \rightarrow \infty} P\left(\frac{\beta(0, \psi)}{\Delta+K} \leq \alpha ; \Psi([a \sqrt{n}],[b n])\right)=\operatorname{ERFC}\left(c \sqrt{\frac{1-x}{2 x}}\right)=\gamma_{c}(\alpha)
$$

with $c=a / \sqrt{b}$. 
Proof: Follows from the Burtin statement [5], item (II), p. 411.

Proposition 1 follows from Corollary 5 and Lemma 5.

\section{Discretizations of the Logistic Mapping}

\subsection{Distribution of Basins for the Logistic Mapping}

Consider the logistic mapping

$$
f(x)=4 x(1-x)=1-(2 x-1)^{2}, x \in[0,1] .
$$

The dynamical system generated by this mapping is a classical example of a chaotic one-dimensional system. Denote by $L_{\nu}$ the uniform $1 / \nu$ lattice on $[0,1]$ :

$$
L_{\nu}=\{0,1 / \nu, 2 / \nu, \ldots, 1\}, \quad \nu=1,2, \ldots
$$

For $x \in[0,1]$ and $k / \nu \leq x<(k+1) / \nu$, for some $0 \leq k \leq \nu-1$, denote the roundoff operator $[x]_{\nu}$ by

$$
[x]_{\nu}=\left\{\begin{array}{cc}
k / \nu, & \text { if } k / \nu \leq x \leq(k+1 / 2) / \nu, \\
(k+1) / \nu, & \text { if }(k+1 / 2) / \nu \leq x \leq(k+1) / \nu .
\end{array}\right.
$$

Denote by $f_{\nu}$ the mapping $L_{\nu} \mapsto L_{\nu}$ defined by

$$
f_{\nu}(\xi)=[f(\xi)]_{\nu}, \quad \xi \in L_{\nu}
$$

The mapping $f_{\nu}$ is a $L_{\nu}$-discretization of the mapping $f$.

For each $\nu$ the set $L_{\nu}$ is partitioned into a disjoint union of basins of attraction of different cycles. Therefore this defines the cardinalities $\mathscr{B}\left(f_{\nu}\right)$. For each $\nu$ and each positive integer $k$ there are defined $k$-sequences $\mathscr{B}_{k}\left(f_{\nu}\right)=\operatorname{ord}_{k}\left(\mathscr{B}_{\mathcal{B}}\left(f_{\nu}\right)\right)$; here, as above, the $i$-th element of $\operatorname{ord}_{k}(\mathscr{B}(\nu))$ is defined to be zero if $i$ is greater than the number of elements in $\mathscr{B}\left(f_{\nu}\right)$.

\section{Principle of Correspondence for Large Basins Distribution}

There exist positive constants $a$ and $b$ such that for large $N$ and $n$, the statistical properties of the distribution of the set

$$
\mathbf{B}_{k}(N, n)=\left\{\mathfrak{B}_{k}\left(f_{N+1}\right), \ldots, \mathfrak{B}_{k}\left(f_{N+n}\right)\right\}
$$

are close to those of the random set

$$
\mathbf{B}_{k}^{*}(N, n)=\left\{\mathfrak{B}_{k}\left(\psi_{N+1}\right), \ldots, \mathfrak{B}_{k}\left(\psi_{N+n}\right)\right\}
$$

where $\psi_{\nu}$ is a random element from the set

$$
\Psi\left(\left[a \frac{\sqrt{\nu}}{\ln (\nu)}\right],\left[b \frac{\nu}{\ln (\nu)}\right]\right)
$$


See [6], p. 562-564 for justification and discussions of this principle. The key parameter $c=a / \sqrt{b}$ was identified in [7] as approximately 0.9. Making use of Corollaries 1, 2 and Proposition 2 above, we can get from this principle the following.

Corollary 6:

(a) For typical large $N$ and $1 \ll n \ll N$, the distribution

$$
D_{\mathscr{B}, 1}(\alpha ; N, n) \triangleq \mathscr{D}\left(\alpha ; \mathbf{B}_{1}(N, n)\right)
$$

is close to the function $F_{\mathscr{B}, 1}(\alpha ; 0.9 / \sqrt{\ln (N)})$.

(b) For typical large $N$ and $1 \ll n \ll N$, the distribution

$$
\left.D_{g_{*}}(\alpha ; N, n) \triangleq \mathscr{D}\left(\alpha ;\left\{g_{*}\left(f_{N}\right), \ldots, g_{*} f_{N+n}\right)\right\}\right)
$$

is close to the function $F_{g_{*}}(\alpha ; 0.9 / \sqrt{\ln (N)})$.

(c) For typical large $N$ and $1{ }^{*} \ll n \ll N$, the function

$$
M_{\mathscr{B}}(\alpha ; N, n) \triangleq \frac{1}{\nu} \sum_{\nu=N}^{N+n} D_{\mathscr{B}}\left(\alpha ; f_{\nu}\right)
$$

is close to the function $F_{\mathscr{B}}(\alpha ; 0.9 / \sqrt{\ln (N)})$.

The above formulated assertion admits to experimental testing. See, for example Figures 6 and 7. A large number of other experiments have also been carried out. All our experiments support strongly the principle of correspondence within the range of several percent.

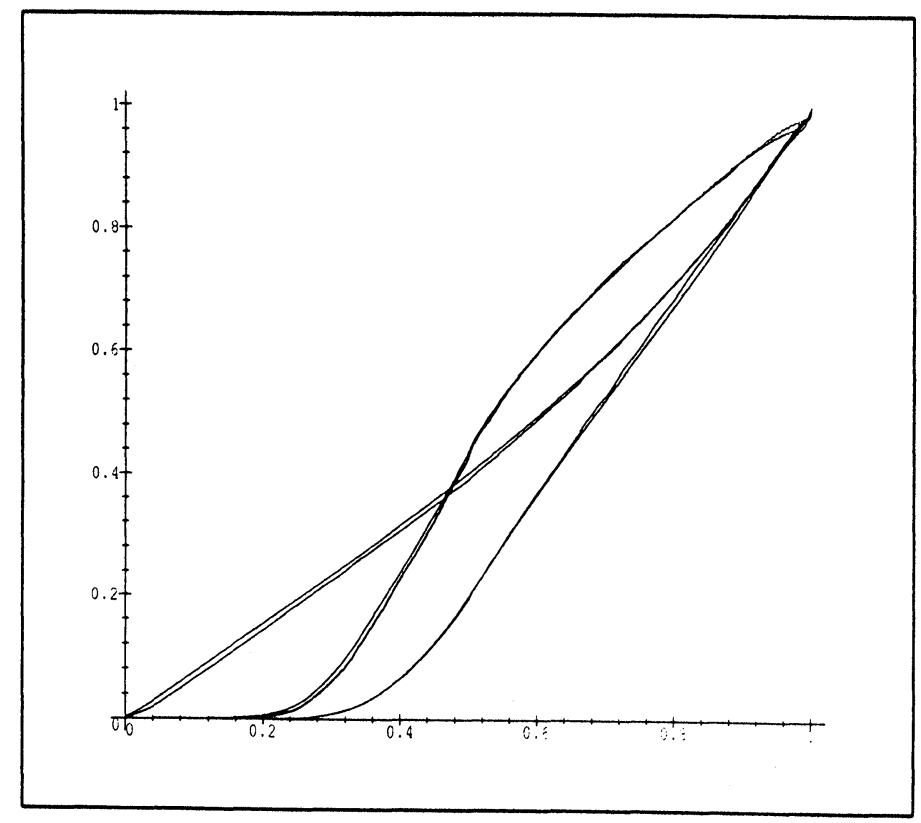

Figure 6. The distributions $D_{\mathbb{C}_{, 1}}\left(\alpha, 10^{5}, 10^{4}\right), D_{g_{*}}\left(\alpha, 10^{5}, 10^{4}\right)$ and the function $M_{\mathscr{B}}\left(\alpha,\left(10^{5}, 10^{4}\right)\right) \quad$ against the theoretical predictions $F_{\mathscr{B}, 1}\left(\alpha, 0.9 / \sqrt{\ln \left(10^{5}\right)}\right)$, $D_{g_{*}}\left(\alpha, 0.9 / \sqrt{\ln \left(10^{5}\right)}\right)$, and $F_{\mathscr{B}}\left(\alpha, 0.9 / \sqrt{\ln \left(10^{5}\right)}\right)$. 


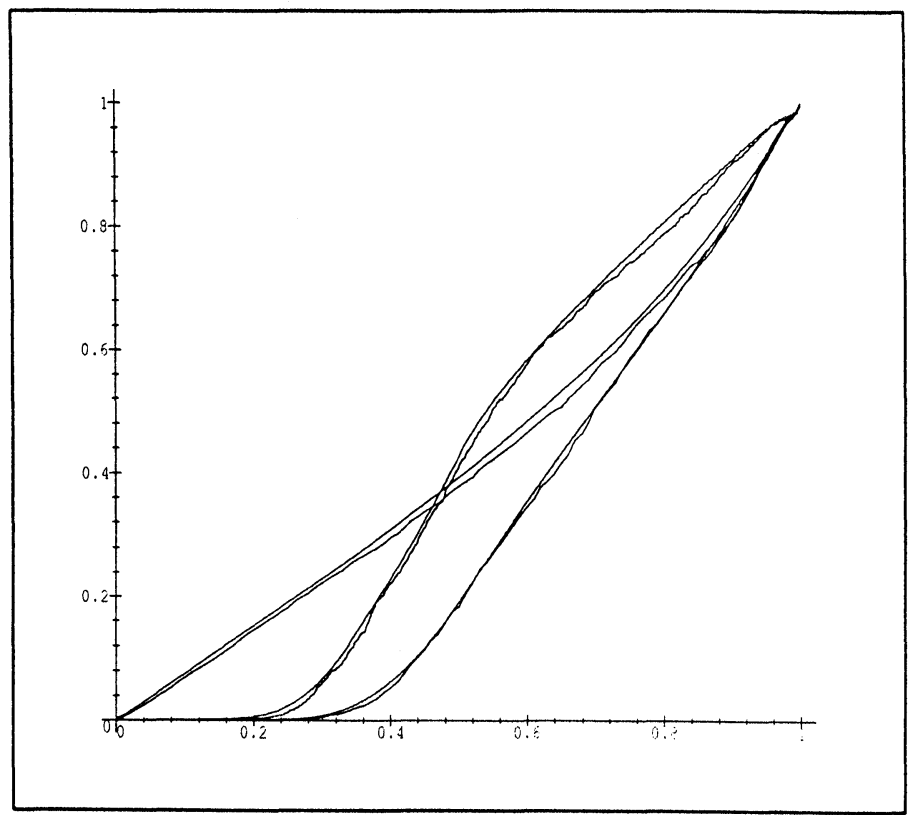

Figure 7. The same as in Figure 6 for $N=10^{7}$ and $n=10^{3}$.

\subsection{Cycles}

For each $\nu$, the set $L_{\nu}$ is partitioned into a disjoint union of basins of attraction of different cycles. Denote by $\mathrm{C}\left(f_{\nu}\right)$ the set of cardinalities of such cycles. For each $\nu$ and each positive integer $k$ there are defined $k$-sequences $\mathfrak{C}_{k}\left(f_{\nu}\right)=\operatorname{ord}_{k}\left(\mathbb{C}\left(f_{\nu}\right)\right)$.

Analogous to the principle of correspondence for large basins distribution, there is:

\section{Principle of Correspondence for Large Cycle Distributions}

For large $N$ and $n$ the statistical properties of distribution of the set

$$
\mathbf{C}_{k}(N, n)=\left\{\mathfrak{C}_{k}\left(f_{N+1}\right), \ldots, \mathcal{C}_{k}\left(f_{N+n}\right)\right\}
$$

are close to those of the random set

$$
\mathbf{C}_{k}^{*}(N, n)=\left\{\mathfrak{C}_{k}\left(\psi_{N+1}\right), \ldots, \mathfrak{C}_{k}\left(\psi_{N+n}\right)\right\}
$$

where $\psi_{\nu}$ is a random element from the set

$$
\Psi\left(\left[a \frac{\sqrt{\nu}}{\ln (\nu)}\right],\left[b \frac{\nu}{\ln (\nu)}\right]\right)
$$


and $a, b$ are the same as in the first principle of correspondence.

The parameter $b$ was identified as approximately 4.45 . Therefore, by Corollary 3 and Proposition 4, we can state the following:

Corollary 7:

(a) For typical large $N$ and $1 \ll n \ll N$, the distribution

$$
D_{\mathrm{C}_{, 1}}(\alpha ; N, n) \triangleq \mathscr{D}\left(\alpha,\left\{\mathrm{e}_{1}\left(f_{\nu}\right) \sqrt{\frac{\ln (\nu)}{\nu}}: N \leq \nu \leq N+n\right\}\right)
$$

is close to the function $F_{\mathrm{C}, 1}^{*}(\sqrt{4.45} \alpha ; 0.9 / \sqrt{\ln (N)})$.

(b) For typical large $N$ and $1 \stackrel{1}{\ll} n \ll$, the function

$$
M_{\mathrm{e}}(\alpha ; N, n) \triangleq \frac{1}{\nu} \sum_{\nu=N}^{N+n} \mathscr{D}\left(\alpha ;\left\{\mathrm{C}\left(\xi, f_{\nu}\right) \sqrt{\frac{\ln (\nu)}{\nu}}: \xi \in L_{\nu}\right\}\right)
$$

is close to the function $F_{\mathrm{e}}^{*}(\sqrt{445} \alpha ; 0.9 / \sqrt{\ln (N)})$.

See Figure 8 for numerical testing at $n=10^{5}, n=10^{4}$. Again, many similar experiments have been carried out.

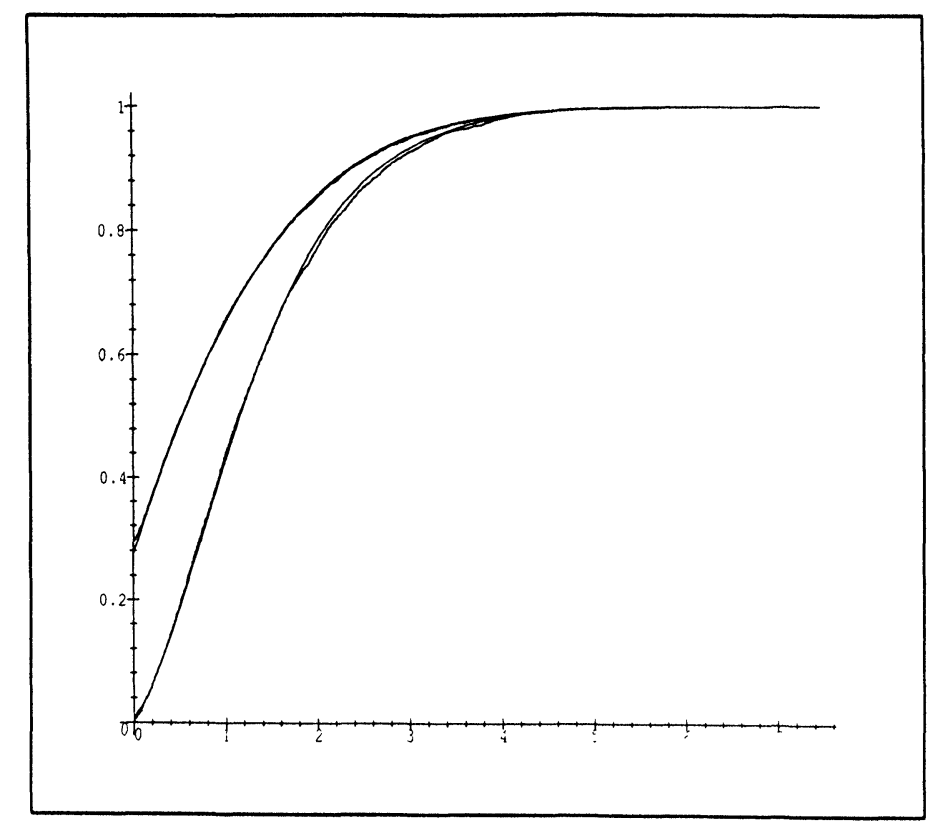

Figure 8. The distributions $D_{\mathrm{C}_{1}}\left(\alpha, 10^{5}, 10^{4}\right), D_{g_{*}}\left(\alpha, 10^{5}, 10^{4}\right)$ and the function $M_{\mathrm{C}}\left(\alpha,\left(10^{5}, 10^{4}\right)\right), F_{\mathrm{C}, 1}^{*}\left(\sqrt{4.45} \alpha, 0.9 / \sqrt{\ln \left(10^{5}\right)}\right)$, and $F_{\mathrm{C}}^{*}\left(\sqrt{4.45} \alpha, 0.9 / \sqrt{\ln \left(10^{5}\right)}\right)$. 


\section{Acknowledgements}

The authors would like to thank Professor Peter Kloeden for useful discussions.

\section{References}

[1] Beck, C., Scaling behavior of random maps, Phys. Lett. A 136:3 (1989), 121125.

[2] Beck, C. and Roepstorff, G., Effects of phase space discretization on the longtime behavior of dynamical systems, Phys. D 25:1-3 (1987), 173-180.

[3] Binder, P.M., Limit cycles in a quadratic discrete iteration, Phys. D 57:1-2 (1992), 31-38.

[4] Bollobas, B., Random Graphs, Academic Press, London 1985.

[5] Burtin, Ju.D., On a simple formula for random mappings and its applications, J. Appl. Probab. 17 (1980), 403-414.

[6] Diamond, P., Kloeden, P., Pokrovskii, A. and Vladimirov, A., Collapsing effects in numerical simulation of a class of chaotic dynamical systems and random mappings with a single attracting center, Phys. D 86 (1995), 559-571.

[7] Diamond, P. and Pokrovskii, A., Statistical laws of computational collapse of discretized chaotic mappings, Intern. J. of Bifurcation and Chaos in Appl. Sci. and Eng., to appear.

[8] Grebogi, C., Ott, E. and Yorke, J.A., Roundoff-induced periodicity and the correlation dimension of chaotic attractors, Phys. Rev. A 34:7 (1988), 36883692 .

[9] Jakobson, M.V., Ergodic theory of one-dimensional mappings, In: Dynamical Systems II Vol. 2 of the Encyclopedia of Mathematical Science (ed. by Ya. G. Sinai) Springer-Verlag, Berlin 199.

[10] Levy, Y.E., Some remarks about computer studies of dynamical systems, Phys. Lett. 88A:1 (1982), 1-3.

[11] de Melo, W. and van Strien, S., One-Dimensional Dynamics, Springer-Verlag, Berlin 1993.

[12] Nusse, H.E. and Yorke, J.A., Is every approximate trajectory of some process near an exact trajectory of a nearby process?, Comm. Math. Phys. 114:3 (1988), 363-379.

[13] Percival, I. and Vivaldi, F., Arithmetical properties of strongly chaotic motions, Phys. D 25:1-3 (1987), 105-130.

[14] Stepanov, V.E., Limit distributions of certain characteristics of random mappings, Theory Prob. Appl. 14 (1969), 612-626.

[15] Stepanov, V.E., Random mappings with a single attracting center, Theory Prob. Appl. 16 (1971), 155-161. 


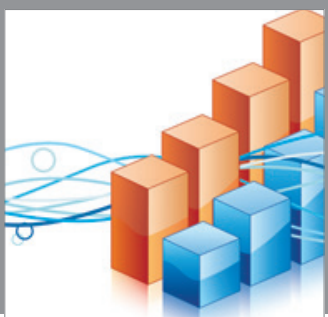

Advances in

Operations Research

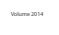

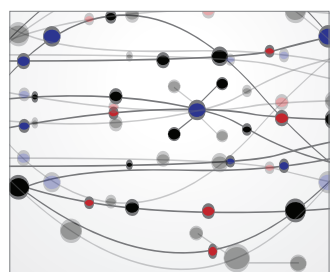

\section{The Scientific} World Journal
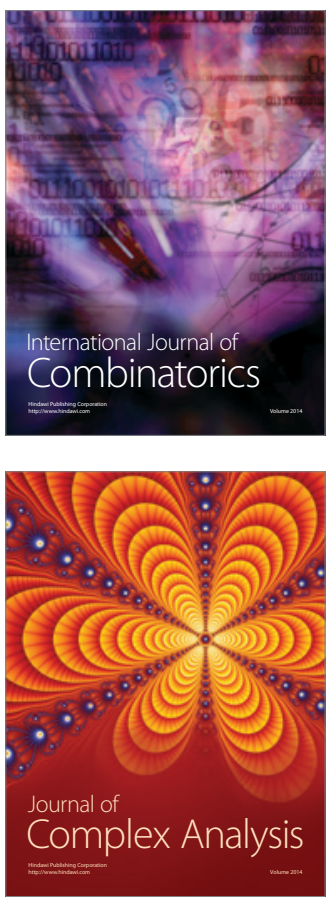

International Journal of

Mathematics and

Mathematical

Sciences
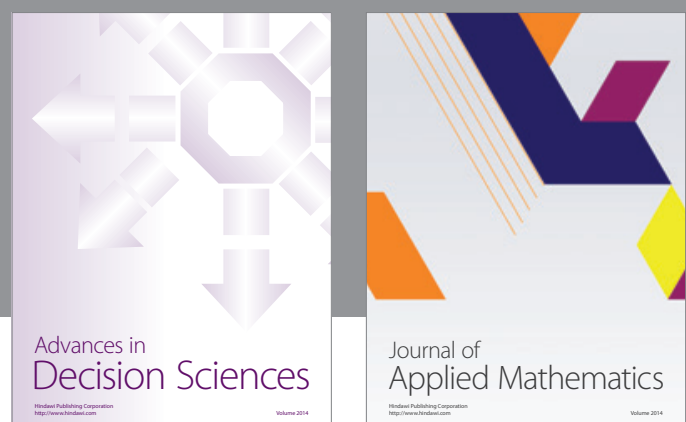

Journal of

Applied Mathematics
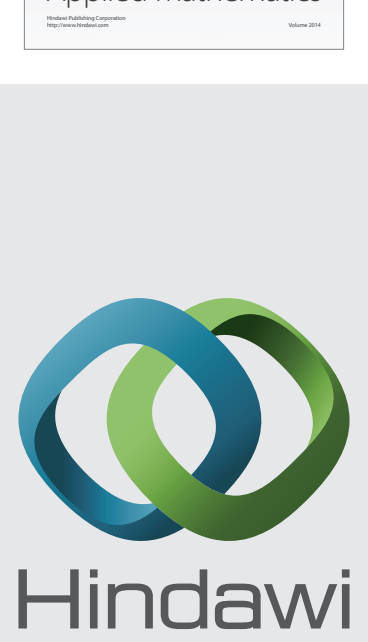

Submit your manuscripts at http://www.hindawi.com
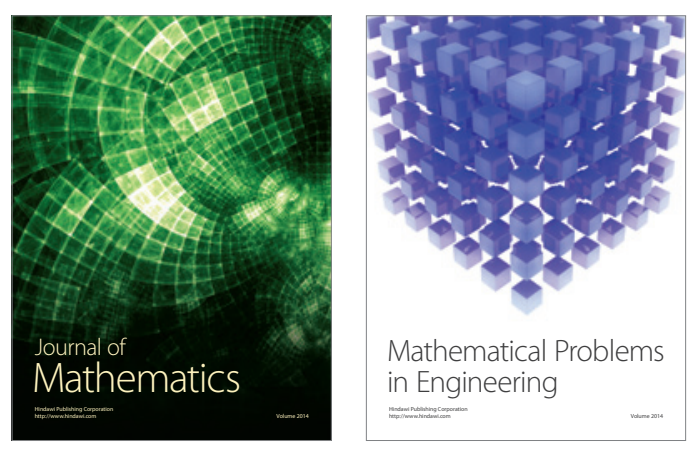

Mathematical Problems in Engineering
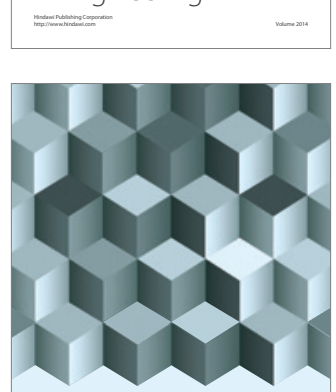

Journal of

Function Spaces
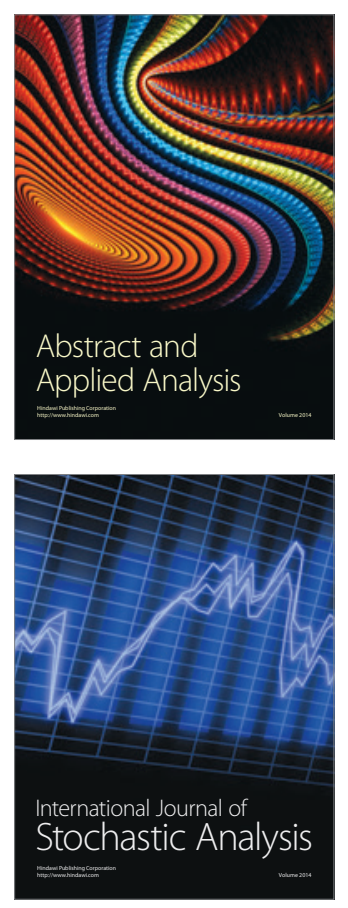

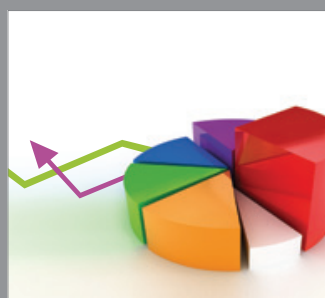

ournal of

Probability and Statistics

Promensencen
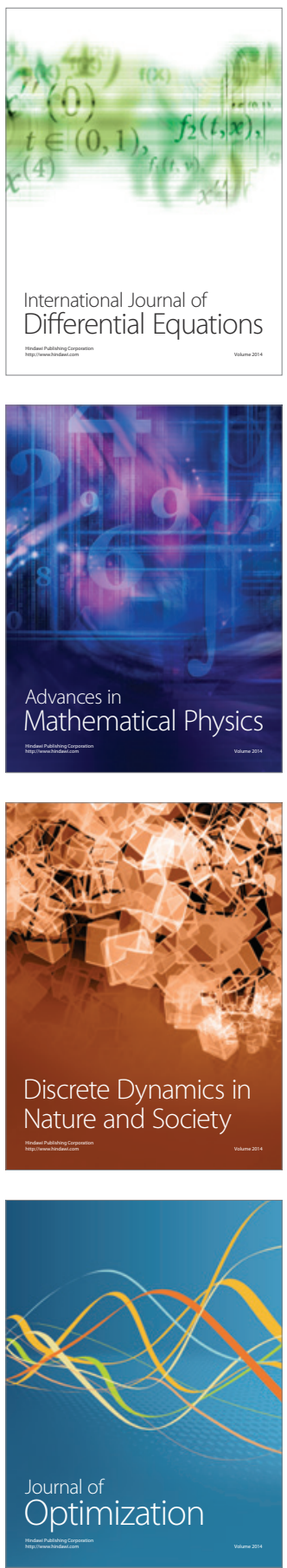